\title{
Singular Translates of Measures on Linear Spaces *
}

By

\author{
R. M. DUDLEY
}

\section{Introduction}

Given a random element $N$ with values in a linear space $S$, and a fixed element $s$ of $S$, we shall give a simple sufficient condition in terms of second moments (Theorem 2 below) for mutual singularity of the distributions of $N$ and $N+s$ : that is, that for some disjoint sets $A$ and $B, N$ and $N+s$ should lie almost surely in $A$ and $B$ respectively. One consequence is a simpler proof of a recent result of V. N. SUDAKov [16] for measures on Hilbert space (see $\S 5$ ).

For random elements which are Gaussian with mean 0 in a natural sense, our condition is necessary and sufficient for singularity, and there is mutual absolute continuity (equivalence) if it fails (Theorem 3 below). This was essentially known; see the end of $\S 6$.

If $N$ is regarded as "noise", then our condition can be interpreted as a way of choosing a "signal" $s$ so that $N$ and $N+s$ can almost certainly be distinguished: see Feidman [6].

Acknowledgments. This paper is based on some work done for the functional integration seminar at Berkeley, spring 1963. I would like to thank the other members of the seminar, especially J. Feldman, for their interest and suggestions.

\section{Random linear functionals}

It is convenient to formulate our results in terms of "weak distributions" or "random linear functionals".

Let $F$ be the field of real or complex numbers, and let $Q$ be any linear space over $F$. Let $Q^{a}$ be the (algebraic) dual space of $Q$, i.e. the linear space of all $F$-linear mappings of $Q$ into $F$. For any $n$-tuple $\left(q_{1}, \ldots, q_{n}\right)$ of members of $Q$ and Borel set $B$ in $n$-space $F^{n}$, let

$$
S\left(q_{1}, \ldots, q_{n}, B\right)=\left\{q^{\prime} \in Q^{a}:<q^{\prime}\left(q_{1}\right), \ldots, q^{\prime}\left(q_{n}\right)>\in B\right\} .
$$

Let $\mathfrak{Q}_{Q}$ be the $\sigma$-algebra of subsets of $Q^{a}$ generated by all such sets.

Definition. A random linear functional (r.l.f.) on $Q$ is an assignment to each $n$-tuple $\left(q_{1}, \ldots, q_{n}\right)$ of members of $Q$ a Borel probability measure $\mu\left(q_{1}, \ldots, q_{n}\right)$ ( ) on $F^{n}$, such that for any $q_{i}, r_{j} \in Q$ and Borel sets $B \subset F^{n}$ and $C \subset F^{m}$,

$$
\begin{gathered}
S\left(q_{1}, \ldots, q_{n}, B\right)=S\left(r_{1}, \ldots, r_{m}, C\right) \\
\text { implies } \\
\mu\left(q_{1}, \ldots, q_{n}\right)(B)=\mu\left(r_{1}, \ldots, r_{m}\right)(C) .
\end{gathered}
$$

* This research was partially supported by National Science Foundation Grant GP-2. 
Note that the condition $S\left(q_{1}, \ldots, q_{n}, B\right)=S\left(r_{1}, \ldots, r_{m}, C\right)$ amounts to a linear relation or set of relations between (some of) the $q_{i}$ and $r_{j}$, with $B$ and $C$ being related in the same way. Thus the involvement of $Q^{a}$ could be avoided. However, for our purposes the condition as given seems to be the simplest.

The following theorem, also (like Theorem 3) essentially known, justifies the name "random linear functional":

Theorem 1. There is a 1-1 correspondence between the set of all r.l.f.'s $\mu($ ) ( ) on a linear space $Q$ and all probability measures $P$ on $\Omega_{Q}$ in $Q^{a}$, given by

$$
P\left(S\left(q_{1}, \ldots, q_{n}, B\right)\right)=\mu\left(q_{1}, \ldots, q_{n}\right)(B) .
$$

Proof. First, if $\mu()()$ is an r.l.f., $P$ is uniquely defined by (*) on sets of the form $S\left(q_{1}, \ldots, q_{n}, B\right)$. Let us show that $P$ extends to a countably additive probability measure on $\mathfrak{Q}_{Q}$. Let $I$ be a (Hamel) basis of $Q$ as a vector space over $F$. Then $Q^{a}$ is realized as the set of all $F$-valued functions on $I$, i. e. the product space

where each $F_{i}$ is isomorphic to $F$.

$$
\prod_{i \in I} F_{i}
$$

For each finite subset $J=\left(q_{1}, \ldots, q_{n}\right)$ of $I$ there is a natural projection $p_{J}$ of $Q^{a}$ onto $G_{J}=\prod_{i \in J} F_{i}$. For each such $J, P_{J}=P \circ p_{J}^{-1}$ is the countably additive Borel measure $\mu\left(q_{1}, \ldots, q_{n}\right)$ on $G_{J}$. If $K$ includes $J$ and $f_{K J}$ is the natural projection of $G_{K}$ onto $G_{J}$, then clearly $P_{K} \circ f_{K J}^{-1}=P_{J}{ }^{\star}$.

Thus we have a family of probability spaces $\left(G_{J}, P_{J}\right)$ with connecting mappings $f_{K J}$ satisfying all the hypotheses of BocHNeR's theorem (BocHNER [2], Theorem 5.1.1 p. 120) on inverse limits of measure spaces: the mappings are continuous, the measures are regular Borel measures and we have "sequential maximality". Thus $P$ extends to a countably additive measure on the generated $\sigma$-algebra in the inverse limit space, which is $Q^{a}$. Since any set $S\left(r_{1}, \ldots, r_{m}, B\right)$ is of the form $S\left(q_{1}, \ldots, q_{n}, C\right)$ for some finite subset $\left(q_{1}, \ldots, q_{n}\right)$ of $I, P$ is defined precisely on $\mathfrak{L}_{Q}$, independently of $I$, since a measure on a ring has at most one countably additive extension to the generated $\sigma$-ring.

Conversely, given a probability measure $P$ on $\Omega_{Q}$ in $Q^{a},\left({ }^{*}\right)$ defines a Borel probability measure $\mu\left(q_{1}, \ldots, q_{n}\right)$ on $F^{n}$ for each $q_{1}, \ldots, q_{n} \in Q$, and the consistency condition is easy to verify, so that $\mu()$ ( ) is a r.l.f., q.e.d.

If $P$ and $\mu()(\quad)$ are related by $\left(^{*}\right)$, we shall call $P$ the distribution of $\mu()()$.

\section{Quadratic r.l.f.'s: the main theorem}

We call a r.I.f. $\mu(\quad)($ ) "quadratic" if

$$
\int|x|^{2} \mu(q)(d x)<+\infty
$$

for each $q \in Q$. If $\mu(\quad)(\quad)$ is quadratic, let

$$
m(q)=\int_{F} x \mu(q)(d x)=\int_{Q^{a}} q^{\prime}(q) d P\left(q^{\prime}\right) .
$$

* The proof can be completed at this point by applying Koumogonov's theorem on product space measures (see M. Lok̀ve, Probability Theory, second edition (Princeton, Van Nostrand, 1960) 4.3 A, p. 93). 
Then clearly $m \in Q^{a}$. We call $m$ the mean of the r.l.f. $\mu($ ) ( ). (Of course the mean is everywhere defined even for certain non-quadratic r.1.f.'s.) We also have a function $B$ on $Q \times Q$ defined by

$$
B(q, r)=\int_{Q^{a}} q^{\prime}(q) \overline{q^{\prime}(r)} d P\left(q^{\prime}\right)=\int_{F} \int_{F} x \bar{y} \mu(q, r)(d x, d y) .
$$

$B(q, r)$ is clearly linear in $q$, conjugate-linear in $r$, nonnegative definite and conjugate-symmetric. Thus we have an "inner product"

$$
(q, r)_{P}=B(q, r)
$$

where $(q, q)_{P}$ may be 0 for $q \neq 0$. It defines a topology on $Q$ by the pseudo-norm $\|q\|_{P}=(q, q)_{P}^{1 / 2}$. We call $B$ the covariance of the r.l.f.

If $z \in Q^{a}$ and $P$ is a probability measure on $\Omega_{Q}$, let $P_{z}$ be the translate of $P$ by $z$ :

$$
P_{z}(A)=P(A-z), \quad A \in \mathfrak{Q}_{Q} .
$$

Here is our condition for singularity of $P$ and $P_{z}$ :

Theorem 2. If $\mu()(\quad)$ is a quadratic r.l.f. on a linear space $Q$ with distribution $P, z \in Q^{a}$, and $z$ is not continuous on $Q$ for \|\|$_{P}$, then $P$ and $P_{z}$ are singular.

Proof. Suppose $z$ is discontinuous. We show first that there are elements $f_{n}$, $n=1,2, \ldots$, of $Q$, orthonormal for $(,)_{P}$, such that $\left|z_{n}\right|=\left|z\left(f_{n}\right)\right|>1$ for all $n$. Since $z$ is discontinuous, there is an $f_{1} \in Q$ such that

$$
\left\|f_{1}\right\|_{P}=1 \text { and }\left|z\left(f_{1}\right)\right|>1 \text {. }
$$

Given $f_{1}, \ldots, f_{n}$, let $p$ be the orthogonal projection of $Q$ onto the subspace $G$ they span ( $p$ is a linear map of $Q$ into itself since $G$ is finite-dimensional). Suppose $p(q)=0$ and $\|q\|_{P}=1$ imply $|z(q)| \leqq 1$. Then for any $h \in Q$,

$$
\begin{aligned}
|z(h)| & =|z(p(h))+z(h-p(h))| \\
& \leqq\left|\sum_{j=1}^{n}\left(h, f_{j}\right)_{P} z\left(f_{j}\right)\right|+\|h-p(h)\|_{P} \\
& \leqq\|h\|_{P}\left(1+\sum_{j=1}^{n}\left|z\left(f_{j}\right)\right|\right),
\end{aligned}
$$

contradicting the fact $z$ is discontinuous. Thus the induction can continue.

Now for any $n$ we have

so that

$$
\int\left|\sum_{j=1}^{n} \overline{z_{j}} q^{\prime}\left(f_{j}\right)\right|^{2} d P\left(q^{\prime}\right)=\sum_{j=1}^{n}\left|z_{j}\right|^{2}=K_{n} \geqq n,
$$

On the other hand,

$$
P\left(q^{\prime}:\left|\sum_{j=1}^{n} \overline{z_{j}} q^{\prime}\left(f_{j}\right)\right| \leqq K_{n}^{3 / 4}\right) \geqq 1-K_{n}^{-1 / 2} .
$$

$$
\begin{aligned}
P_{z}\left(q^{\prime}:\left|\sum_{j=1}^{n} \overline{z_{j}} q^{\prime}\left(f_{j}\right)\right|\right. & \left.\leqq K_{n}^{3 / 4}\right)=P\left(q^{\prime}:\left|\sum_{j=1}^{n} \overline{z_{j}}\left(q^{\prime}\left(f_{j}\right)+z_{j}\right)\right| \leqq K_{n}^{3 / 4}\right) \\
& =P\left(q^{\prime}:\left|\left(\sum_{j=1}^{n} \overline{z_{j}} q^{\prime}\left(f_{j}\right)\right)+K_{n}\right| \leqq K_{n}^{3 / 4}\right) \leqq \\
& \leqq P\left(q^{\prime}:\left|\sum_{j=1}^{n} \overline{z_{j}} q^{\prime}\left(f_{j}\right)\right| \geqq K_{n}-K_{n}^{3 / 4}\right) .
\end{aligned}
$$


For $n \geqq 16, K_{n}-K_{n}^{3 / 4} \geqq K_{n} / 2$, so that the latter $P$-measure is at most $4 / K_{n}$ by the same mean-square argument as above. Thus we have defined a sequence of sets $A_{n}$ such that $P\left(A_{n}\right) \rightarrow 1$ and $P_{z}\left(A_{n}\right) \rightarrow 0$ as $n \rightarrow \infty$. Hence $P$ and $P_{z}$ are singular. (If $B_{m}=A_{2 m}$, and $B$ is the set of points belonging to infinitely many $B_{m}$, then $P(B)=1$ and $P_{z}(B)=0$.) This completes the proof.

\section{Distributions on topological linear spaces}

If $Q$ is a topological linear space, it is natural to consider r.l.f.'s $\mu()(\quad)$ which are continuous in the sense that $q \rightarrow \mu(q)$ is continuous with respect to the topology of weak convergence of measures on $F$. If $Q$ is a countably-normed nuclear space, then the topological dual space $Q^{\prime}$ of $Q$ has outer measure 1 for the distribution $P$ of $\mu($ ) ( ) (see Minlos [11] or Gelfard and Vumenkin [7] for details, and KoLmogonov [10] for the shortest proof). If $Q$ is a separable infinite-dimensional Hilbert space, then $Q^{\prime}$ need not have outer measure 1, e.g. for the "normal" Gaussian r.l.f. with mean 0 and covariance equal to the given inner product.

In any case, Theorem 2 implies that if a quadratic r.l.f. with distribution $P$, on a topological linear space $Q$, has a continuous covariance, $P$ and $P_{z}$ are singular for $z$ not in $Q^{\prime}$.

On certain spaces where category arguments are available, a continuous quadratic r.l.f. has a continuous covariance: see e.g. DUdLEY [4] $\$ 4$ Lemma 2. However, for other spaces, e.g. an inner product space of countably infinite Hamel dimension, this is not true.

Likewise, for suitable topological linear spaces the set of members of $Q^{a}$ which are Borel measurable as functions on $Q$ is exactly $Q^{\prime}$. A proof for Banach spaces was given by BaNach [1], Chap. V, § 1 Thme. 1 p. 78.

Now suppose $\mu$ is a Borel probability measure on a topological linear space $S$, and let $Q_{\mu}$ be the set of elements $q$ of $S^{\prime}$ for which

$$
\int|q(s)|^{2} d \mu(s)<+\infty \text {. }
$$

If $S$ is a normed space, we can let

$$
\nu(A)=\int_{A} e^{-\|x\|} d \mu(x) / \int_{S} e^{-\|x\|} d \mu(x)
$$

to obtain a Borel probability measure $v$ such that $\mu_{z}$ and $v_{z}$ are equivalent for all $z$ in $S$, and such that $Q_{v}$ is all of $S^{\prime}$.

In any case, $Q_{\mu}$ is a linear subspace of $S^{\prime}$, and $\mu$ defines a quadratic r.l.f. on $Q_{\mu}$ by

$$
\mu\left(q_{1}, \ldots, q_{n}\right)(B)=\mu\left(s:\left(q_{1}(s), \ldots, q_{n}(s)\right) \in B\right) .
$$

We now have a

Corollary. If $\mu$ is a Borel probability measure on a topological linear space $S$, $z \in S$, and $z$ is discontinuous on $Q_{\mu}$ for $(,)_{\mu}$, then $\mu$ and $\mu_{z}$ are singular.

Proof. $S$ is naturally a subspace of the dual space $\left(Q_{\mu}\right)^{a}$, and we have by Theorem 2 that there is a set $B$ in the $\sigma$-algebra $\Omega_{Q_{\mu}}$ such that $P(B)=1$ and $P_{z}(B)=0$ where $P$ is the distribution of the r.l.f. on $Q_{\mu}$ defined by $\mu$. But 
$B \cap S$ is a Borel set in $S, S$ has $P$-outer measure 1 and $P$ reduced to $S$ is $\mu$, so q.e.d.

$$
\begin{aligned}
\mu(B \cap S) & =P(B)=1, \quad \mu_{z}(B \cap S)=1-\mu_{z}(S \sim(B \cap S)) \\
& =1-P_{z}\left(\left(Q_{\mu}\right)^{a} \sim B\right)=0,
\end{aligned}
$$

If $S$ is a complete separable metric linear space, and $P$ is a Borel probability measure on $S$, then $P$ is concentrated in a countable union $M$ of compact sets, and obviously $P$ and $P_{z}$ are singular for $z$ not in $M-M$. If $S$ is infinite dimensional, then $M-M$ is of first category and so has dense complement. For further information of this type see Sudakov [14] and GiRsanov and Mityagra [8].

\section{Hilbert spaces}

Let $H$ be a separable Hilbert space with inner product ( , ) and $\mu$ a Borel probability measure on $H$. As indicated in $\S 4$, we may assume $s \rightarrow(s, s)$ is integrable for $\mu$, so that $Q_{\mu}$ is the whole topological dual space $H^{\prime}$ of $H$, replacing $\mu$ by an equivalent measure if necessary. As usual we identify $H^{\prime}$ with $H$ by

$$
f \leftrightarrow f^{\prime}:(x, f)=f^{\prime}(x), \quad f \in H, \quad f^{\prime} \in H^{\prime} .
$$

Now, $(,)_{\mu}$ as defined in $\S 4$ is a nonnegative definite, continuous, conjugate-symmetric sesquilinear form on $H$, so we have

$$
(x, y)_{\mu}=(A x, A y)
$$

for some nonnegative, bounded, symmetric (self-adjoint) operator $A$ on $H$. If $\left\{f_{n}\right\}$ is an orthonormal basis of $H$, then

$$
\sum_{n=1}^{\infty}\left(A f_{n}, A f_{n}\right)=\int_{H} \sum_{n}\left|\left(f_{n}, s\right)\right|^{2} d \mu(s)=\int_{H}(s, s) d \mu(s)<\infty .
$$

Thus $A$ is of Hilbert-Schmidt type, and there exists a complete orthonormal set $\left\{g_{n}\right\}$ such that $A\left(g_{n}\right)=\lambda_{n} g_{n}, n=1,2, \ldots$, where

$$
\lambda_{n} \geqq 0, \quad \sum_{n=1}^{\infty} \lambda_{n}^{2}<\infty
$$

According to the Corollary in $\S 4, \mu$ and $\mu_{z}$ are singular if the linear functional $s \rightarrow(s, z)$ is not continuous for $(,)_{\mu}$. But we have

Lemma 1. If $A$ is a bounded operator on a Hilbert space $H$ then $|(s, z)| \leqq$ $\leqq K\|A s\|$ for some $K>0$ and all $s \in H$ if and only if $z$ is in the range of the transpose $A^{*}$ of $A$ (i.e. the operator $A^{*}$ with $(A x, y)=\left(x, A^{*} y\right)$ for all $\left.x, y \in H\right)$.

Proof. If $z=A^{*} w$ for some $w \in H$, then

for all $s$ in $H$.

$$
|(s, z)|=\left|\left(s, A^{*} w\right)\right|=|(A s, w)| \leqq\|w\|\|A s\|
$$

Conversely, if $|(s, z)| \leqq K\|A s\|$ for some $K>0$ and all $s$, then if $A s=0$, $(s, z)=0$. Thus for some linear functional $\psi$ on the range of $A,(s, z)=\psi(A s)$, with $|\psi(x)| \leqq K\|x\|$ for all $x$ in the range of $A$. Thus $\psi(x)=(x, w)$ for some $w \in H$, and $(s, z)=(A s, w)=\left(s, A^{*} w\right)$ for all $s$, so $z=A^{*} w$, q.e.d. 
In our case, $A$ is symmetric, i.e. $A=A^{*}$. Since $A$ is a compact operator, its range is included in a countable union of compact sets, so that we reobtain the result mentioned at the end of $\S 4$.

Sudakov [15], [16] has proved for real Hilbert spaces that if $C$ is a closed, convex, symmetric set such that $\mu$ and $\mu_{z}$ are equivalent for all $z$ in $C$, then

$$
C=G+W
$$

where $G$ is a finite-dimensional subspace and $W$ is a "Schmidt set", i.e. $W$ is the image of a bounded set for some Hilbert-Schmidt operator. We shall reobtain this result with the additional information that $A$ can be taken as the HilbertSchmidt operator for $W$. To include the case of complex Hilbert spaces, we assume $C$ is "circled", i.e. $x \in C$ and $|\lambda|=1, \lambda \in F$, imply $\lambda x \in C$.

Lemma 2. Let $S$ and $T$ be Banach spaces, $A$ a compact operator from $S$ to $T$, and $C$ a closed, convex, circled subset of the range of $A$. Then for some bounded set $M \subset S, C=G+A(M)$ where $G$ is a finite-dimensional subspace.

Proof. We may assume that $A$ is $1-1$ and has dense range (passing to a quotient space or closure respectively if necessary) so that $S$ and $T$ are separable. Let $G$ be the set of $x \in T$ such that $a x \in C$ for all $a>0$. Then since $C$ is convex and circled, $G$ is a vector subspace of $T$, closed since $C$ is closed. Thus since $C$ is included in a countable union of compact sets, $G$ is finite-dimensional by category.

If $f \in G$ and $x \in C$ then $(n-1) x / n+n f / n$ belongs to $C$ for all $n$, so $x+f \in C$. Thus $C$ is a union of cosets (translates) of $G$. Passing to the quotient space $T / G$, we may assume $G=\{0\}$, and wish to prove $C$ is of the form $A(M), M$ bounded.

For $n=1,2, \ldots$, let

$$
C_{n}=\{x \in C:\|x\| \leqq n\}, \text { and }\|x\|_{n}=\inf \left\{a>0: x \in a C_{n}\right\} .
$$

Then \|\|$_{n}$ is a norm with unit ball $C_{n}$ on the linear subspace

$$
T_{n}=\bigcup_{a>0} a C_{n}
$$

Now suppose $\left\{x_{r}\right\}$ is a Cauchy sequence in $T_{n}$ for \|\|$_{n}$. Then for some $m>0$, $x_{r} \in m C_{n}$ for all $r$, and $x_{r}$ converges for the original norm on $T$ to some $x$, with $x \in m C_{n}$ since $m C_{n}$ is closed. For any $\varepsilon>0$ there is a $k$ such that $x_{r}-x_{s} \in \varepsilon C_{n}$ for $r, s \geqq k$, so $x_{r}-x \in \varepsilon C_{n}$ for $r \geqq k$ since $\varepsilon C_{n}$ is closed, and

$$
\lim _{r \rightarrow \infty}\left\|x_{r}-x\right\|_{n}=0 \text {. }
$$

Thus $\left(T_{n},\|\|_{n}\right)$ is a Banach space.

Now suppose $u_{r} \rightarrow u$ in $S$ and $A\left(u_{r}\right) \rightarrow x$ in $\left(T_{n},\|\| n\right)$. Then $A\left(u_{r}\right) \rightarrow A(u)$ for the given norm on $T$, so $A(u)=x$ since $A\left(u_{r}\right) \rightarrow x$. Thus $A^{-1}$ is a closed operator from all of $\left(T_{n},\|\|_{n}\right)$ to $S$, thus bounded. Hence for each $n, A^{-1}\left(C_{n}\right)$ is bounded in $S$, and $C_{n}$ is compact (since it is closed).

Now if $C$ is not bounded, there are $y_{m} \in C$ with $\left\|y_{m}\right\|=m, m=1,2, \ldots$. Then $y_{m} / m \in C_{1}$ for all $m$, so the sequence $y_{m} / m$ has a subsequence convergent to some $y \in C_{1}$ with $\|y\|=1$. Now for any $n>0, n y=\lim _{m \rightarrow \infty} n y_{m} / m \in C$, taking the limit along our subsequence, so $y \in G$, a contradiction since $y \neq 0$. Thus $C=C_{n}$ for some $n$ and $A^{-1}(C)$ is bounded, q.e.d. 


\section{Gaussian distributions}

The converse of Theorem 2 is false in general. For example, if $Q$ is one-dimensional and $P$ is an atomic measure concentrated in the point $v \neq 0$, then any $w \in Q^{\prime}$ is continuous for the covariance but $P$ and $P_{w}$ are singular for $w \neq 0$. However, the converse holds for certain Gaussian r.l.f.'s (an r.l.f. $\mu()($ ) is Gaussian if each $\mu\left(q_{1}, \ldots, q_{n}\right)$ is a (possibly degenerate) Gaussian measure on $n$-space).

Theorem 3. If $P$ is the distribution on $Q^{a}$ of a Gaussian r.l.f. with mean 0 on a linear space $Q$, then $P$ and $P_{z}$ are equivalent if $z$ is continuous for the covariance. $B$ of the r.l.f.

Proof. It suffices to show that $P_{z}$ is absolutely continuous with respect to $P$. Suppose there is a set $A$ in $\Omega_{Q}$ such that $P(A)=0, P_{z}(A)>0$. Then there is a countable set $q_{1}, q_{2}, \ldots$, of elements of $Q$ such that $A$ is in the $\sigma$-algebra generated by the sets of the form $S\left(q_{n}, C\right), n=1,2, \ldots$. We may assume that the $q_{n}$ are linearly independent and then that they are orthogonal for $B$ (by the Gram-Schmidt process).

Now if $B\left(q_{j}, q_{j}\right)=0$ for some $j$, then $z\left(q_{j}\right)=0$ by continuity and sets of the form $S\left(q_{j}, C\right)$ have measure 1 or 0 according as $0 \in C$ or not, both for $P$ and $P_{z}$. Thus, replacing those of measure 1 by all of $Q^{a}$ and those of measure 0 by the null set, we obtain a set $A^{\prime}$ in the $\sigma$-ring generated by the sets $S\left(q_{n}, C\right)$ for $n=j$ with $P_{z}\left(A^{\prime}\right)=P_{z}(A), P\left(A^{\prime}\right)=0$. Thus we may assume $B\left(q_{j}, q_{j}\right)>0$ for all $j$ and normalize to $B\left(q_{j}, q_{j}\right)=1$.

Now $\left\{q_{j}\right\}$ is an orthonormal set for $B$ and we have

$$
\sum_{j=1}^{\infty}\left|z_{j}\right|^{2}<\infty
$$

where $z_{j}=z\left(q_{j}\right)$. From here on we are, in a sense, only proving the known fact that the normal distribution is quasi-invariant: see SEGAL [13], Theorem 3 p. 22.

Let $f$ be the map of $Q^{a}$ onto a countable product $S$ of copies of $F$ defined by $f(x)=\left\{x_{j}\right\}_{j=1}^{\infty}$ where $x_{j}=x\left(q_{j}\right)$. Then we obtain Borel measures $P^{\prime}=P \circ f^{-1}$ and $P_{z}^{\prime}=P_{z} \circ f^{-1}$ on $S$ such that $P^{\prime}(f(A))=0, P_{z}^{\prime}(f(A))>0$. Let us show that actually $P_{z}^{\prime}$ is absolutely continuous with respect to $P^{\prime}$.

Since our r.l.f. has mean 0 and the $q_{j}$ are $B$-orthonormal, $P^{\prime}$ is a product of Gaussian measures on $F$ with mean 0 and variance 1 , and $P_{z}^{\prime}$ a product of Gaussian measures with mean $z_{j}$ and variance 1 . Thus a Radon-Nikodym derivative of $P_{z}^{\prime}$ with respect to $P^{\prime}$ is given by the infinite product

$$
\prod_{j=1}^{\infty} \exp \left(-z_{j} x_{j}+z_{j}^{2} / 2\right)
$$

if it converges, i. e. if the sum

$$
\sum_{j=1}^{\infty} z_{j} x_{j}-z_{j}^{2} / 2
$$

converges. But $z_{j}^{2} / 2$ is a convergent series of constants and $\sum_{j=1}^{\infty} z_{j} x_{j}$ converges almost everywhere with respect to $P^{\prime}$ since $z_{j} x_{j}$ are independent Gaussian random variables with mean 0 and variance $z_{j}^{2}$. Thus $P_{z}^{\prime}$ is absolutely continuous with respect to $P^{\prime}$, and we have reached a contradiction, completing the proof. 
The assumption that the r.l.f. has mean 0 in Theorem 3 can be weakened to the assumption that the mean $m$ is itself continuous for the covariance of the r.l.f. defined by $P_{-m}$ (it is automatically continuous for that of $P$ ). If not, however, then $P$ and $P_{-m}$ are singular by Theorem 2 .

Other forms of Theorem 3 have appeared in Chover and Feldman [3], FELDman [6], and Gelfand and Vilenkin [7], Chap. IV, §5 Teorema 3.

\section{Random processes}

It is convenient for many purposes to consider not only ordinary stochastic processes $x(t, \omega)$ but r.l.f.'s on the space $\mathfrak{D}$ of complex-valued $C^{\infty}$ functions with compact support on the real line. A process $x(t, \omega)$ whose sample functions ( $\omega$ fixed) are almost all locally integrable defines such a r.l.f. with $\mu\left(f_{1}, \ldots, f_{n}\right)$ being the joint distribution of

$$
\int x(t, \omega) f_{j}(t) d t, \quad j=1, \ldots, n .
$$

If an r.l.f. on $\mathfrak{D}$ is continuous for the usual topology of $\mathfrak{D}$ (ScHwaRTz [12]), we call it a random distribution (r.d.). It is known that the distribution $P$ on $\mathfrak{D}^{a}$ of an r.d. gives outer measure 1 to the topological dual space $\mathfrak{D}^{\prime}$ of $\mathfrak{D}$ (see GELFand and VILENkin [7], Glava IV, $\S 2$, No.4, Teorema 6). Thus $P$ defines a Borel probability measure on $\mathfrak{D}^{\prime}$ which we shall also call $P$.

For any quadratic r.d. the covariance $B(, \quad)$ is a hypo-continuous bilinear function on $\mathfrak{D}$ (see DUDLEY [4], Lemma 2). Thus according to the "Theorem of kernels" (EhrEnPREIS [5], GELFAND and VIlEnkin [7], Chap. 1, § 1, No. 3, Teorema 5, p. 32) there is a distribution $B_{2}$ on the plane such that

$$
B(f, g)=B_{2}(f \otimes \tilde{g})
$$

where $(f \otimes \bar{g})(s, t)=f(s) \bar{g}(t)$. Here $B_{2}$ is conjugate-symmetric under interchange of $s$ and $t$. We call $B_{2}$ the covariance distribution of the r.d.

Thus we can say that $P$ and $P_{z}$ are singular if $z$ is discontinuous for the inner product on $\mathfrak{D}$ defined by $B_{2}$. For a stochastic process $x(t, \omega)$ with locally integrable sample functions, $B_{2}$ is the usual covariance function

$$
K(s, t)=E(x(s) \overline{x(t)}) .
$$

Given a non-quadratic r.d. with distribution $P$, we would like to replace $P$ by an equivalent measure on $\mathfrak{D}^{\prime}$ defining a quadratic r.d. However, this is not always possible: for example, let $P$ give positive mass to the $\delta$-distribution (unit mass at 0 in the real line) and all its derivatives in the sense of distributions. Then since there is no limit to the order of growth of the derivatives of a $C^{\infty}$ function at 0 , no measure equivalent to $P$ yields a quadratic r.d.

On the other hand, if $P$ is concentrated in a subspace of $\mathfrak{D}^{\prime}$ whose relative topology is normizable, we can define an equivalent quadratic r.d. as in $\S 4$.

\section{Stationary processes}

An r.d. is called stationary if $\mu\left(f_{1}, \ldots, f_{n}\right)=\mu\left(g_{1}, \ldots, g_{n}\right)$ whenever there is a real number $b$ such that $f_{i}(x+b)=g_{i}(x), i=1, \ldots, n, f_{1}, \ldots, f_{n} \in \mathfrak{D}$. Such processes have been discussed by ITo [9] and DuDLEY [4]. 
For a quadratic stationary r.d. the covariance distribution $B_{2}$ is unchanged by the transformation $(s, t) \rightarrow(s+h, t+h)$ for any real $h$. Thus (see Schwartz [12], tome I, Ch. II, $\S 6$, Thme. VI) there is a distribution $C$ on the real line such that for any $\psi \in \mathbb{D}\left(R^{2}\right)$,

Thus

$$
B_{2}(\psi)=C_{(x)}\left[\int_{-\infty}^{\infty} \psi(x+h, h) d h\right] .
$$

$$
B(f, g)=C_{(x)}\left[\int_{-\infty}^{\infty} f(x+h) \bar{g}(h) d h\right]
$$

The integral can be interpreted as a convolution $f^{*} \breve{g}$ where $\breve{g}(h)=g(-h)$. It is clear that $C$ is a "positive definite" distribution and hence is the (inverse) Fourier transform of a nonnegative Borel measure $m$ with

$$
m([-x, x]) \leqq K\left(1+x^{2}\right)^{n}
$$

for some positive constants $K$ and $n$ (Schwartz [12], tome II, Chap. VII, $\S 9$, Théorème XVIII, p. 132). Thus we have

$$
B(f, g)=\int_{-\infty}^{\infty} \tilde{f}(k) \bar{g}(k) d m(k)
$$

where $\tilde{f}$ and $\tilde{g}$ are the Fourier transforms of $f$ and $g$ respectively. This form of $B$ was also obtained by K. Iro [9] using another method.

Now suppose that $P$ and $P_{z}$ are not singular for some $z \in \mathfrak{D}^{\prime}$. Then according to Theorem $2, z \in \subseteq^{\prime}(R)$ ( $z$ is a tempered distribution) and we have

$$
z(\varphi)=\int \tilde{\varphi} \eta d m
$$

for some function $\eta$ with $\int|\eta|^{2} d m<\infty$. (The fact that $z \in \widehat{S}^{\prime}$ also follows from the fact that $P$ is concentrated in $\varsigma^{\prime}$ according to part of the main theorem of DUduey [4]). Thus $z=(\eta m)^{\sim}$ where $\eta m$ is the complex-valued Borel measure, finite on compact sets, defined by

$$
(\eta m)(B)=\int_{B} \eta d m
$$

This shows that the spectrum of $z$ is included in that of $m$, and also gives information on the local behavior of $z$ according to the growth of $m$ at $\infty$. We will not try to give more precise information at present.

\section{Reierences}

[1] Banach, S.: Théorie des Opérations Lineaires (Monografje Matematyezne, Warszawa, 1932).

[2] Bochner, S.: Harmonic Analysis and the Theory of Probability. Berkeley and Los Angeles: University of California Press 1955.

[3] Chover, J., and J. FkLdman: On positive definite integral kernels and a related quadratic form. Trans. Amer. math. Soc. 89, 92-99 (1958).

[4] DUdLey, R. M. : Fourier analysis of substationary processes with a finite moment. To appear in Trans. Amer. math. Soc.

[5] Enrenpreis, L.: On the theory of kerrels of Schwartz. Proc. Amer. math. Soc. 7, $713-718(1956)$. 
[6] Felpman, J.: Equivalence and perpendicularity of Gaussian processes. Pacific J. Math. 8, 699-708 (1958).

[7] Gellfand, I. M., and N. Ya. Vilenkin: Generalized Random Processes - Extended Hilbert Spaces (Generalized Functions, vol. 4) (in Russian). Moscow: Fizmatgiz 1962.

[8] GTRsanov, I. V., and B.S. MtTyagin: Quasi-invariance of measures on topological linear spaces (in Russian). Naučn. Doklady vysš. Školy (fiz.-mat. Nauki) 1959, No. 2 pp. 5-9 (1960).

[9] Iто, K.: Stationary random distributions. Mem. Coll. Sci., Univ. Kyoto Ser. A 28, $212-213$ (1953).

[10] Kolmogorov, A. N.: Note to the papers of R. A. Minlos and V. Sazonov (in Russian). Teor. Verojatn. Primen. 4, 237-239 (1959). English translation: Theory Probability Appl. (USSR) 4, 221-223.

[11] Mrnuos, R. A.: Generalized random processes and their extension to measures (in Russian). Trudy Moskovsk. mat. Obšč. 8, 497-518 (1959). English translation: Selected Translations in Mathematical Statistics and Probability 3, 291-314 (1963).

[12] Scrwartz, L.: Théorie des Distributions, second ed. Paris: Hermann 1957 (tome I), 1959 (tome II).

[13] Stgat, I. E.: Distributions in Hilbert space and canonical systems of operators. Trans. Amer. math. Soc. 88, 12-41 (1958).

[14] Suddkov, V. N.: Linear sets with quasi-invariant measure (in Russian). Doklady Akad. Nauk SSSR, n. Ser. 127, 524-525 (1959).

[15] - On a class of compacts in Hilbert space (in Russian). Uspechi mat. Nauk 18, 181-187 (1963).

[16] - On the characterization of quasi-invariance of measures in Hilbert space (in Russian). ibid. 188-190.

Department of Mathematics University of California Berkeley 4, California (USA)

(Received August 14, 1963) 